\title{
Clostridium difficile Infection in Western Romania: Correlations Between Ribotype and Clinical Form One year observational retrospective study
}

\author{
ADELINA RALUCA MARINESCU*, VOICHITA LAZUREANU, RUXANDRA LAZA, VIRGIL MUSTA, NARCISA NICOLESCU, \\ MONICA LICKER \\ University of Medicine and Pharmacy Victor Babes, 2 Eftimie Murgu Sq., 300041, Timisoara, Romania
}

Clostridium difficile is a globally significantenteric pathogen with rapid emergence in the USA, Asia, Oceania and Europe. It is reported to be the leading cause of infectious diarrhea in healthcare facilities of developed nations, and the burden of disease caused by this pathogen is receiving increasing recognition. The objectives of the present study consisted in determining the incidence of the $C$. difficile infection during the last year, correlating the ribotype with the clinical form of disease, as well as the quantification of the importance of the ATLAS score in the evolution of enterocolitis with C. difficile. Also, the secondary objectives of the study were: observation of the pathogens circulation in cases transferred from other hospital units and correlation of the current treatment versus existing antimicrobial guidelines. We have performed an observational retrospective study of Clostridium difficile enterocolitis cases in the Hospital of Infectious Diseases and Pneumophtisiology Victor Babes Timisoara, during January 2018- December 2018. Between January and December 2018, 207 patients with C. difficile acute enterocolitis were hospitalized. The Atlas scores remain of great utility in determining the prevalence of the infection. Of the 207 hospitalized patients in 18 cases, ribotyping was positive. All the 18 ribotypes were with binary toxin (cdt) and genotype 027, a strain commonly found in the west of the country, but also in countries with neighboring borders. Compared to the previous years, there is an alarming increase in the number of cases with $C$. difficile infection, ribotype 027. These isolates had another characteristic that no doubt contributed to their ability to spread quickly in a healthcare environment, they produced very high levels of spores compared to other $\mathrm{C}$. difficile strains.

Keywords: Clostridium difficile, ribotype, clinical form

Over the last decade, there have been a number of changes in the epidemiology, clinical manifestations and development of $C$. difficile infection.

The pathogenicity of $C$. difficile is mainly mediated by the release of two toxins: toxin $A$ and $B$. These large toxins (TcdA, $308 \mathrm{kDa}, T c d B, 270 \mathrm{kDa}$ ) function as glucoziltransferases that inactivate small GTPases, such as Rho, Rac and $\mathrm{Cdc} 42$ in eukaryotic target cells. Highlighting the ribotype in this pathology becomes a prime consideration both by increasing the success rate in healing the disease, reducing the risk of relapse, and by targeting rather than empirically administering the antibiotic [1-4].

According to the previous studies, the prevalence rate of $C$. difficilein the intestinal microbiota of healthyadults is estimated at $0-17 \%$. Recent studies have suggested, however, that the $C$. difficile toxins may be present more frequently (53.3\%) than previously estimated [5].

C. difficile does not cause any significant disease when present in small numbers. Disturbance of the normal intestinal flora - dysbiosis - can lead to unconstrained $C$. difficile extension within the microbiota, which causes inflammation and damage to the intestinal mucosa $[6,7]$.

The objective of the present study consisted in determining the incidence of the $C$. difficileinfection during the lastyear, correlating the ribotype with the clinical form of disease, as well as the quantification of the importance of the ATLAS score in the evolution of enterocolitis with $C$. difficile. Also, the secondary objectives of the study were: observation of the pathogens circulation in cases transferred from other hospital units and correlation of the current treatment versus existing antimicrobial guidelines.

\section{Experimental part}

We have performed an observational retrospective study of $C$. difficile enterocolitis cases in the Hospital of Infectious Diseases and Pneumophtisiology Victor Babes Timisoara, during J anuary 2018- December 2018. All the data comes from patients admitted to this hospital, which is composed of two departments of infectious diseases.

We only included laboratory confirmed cases in patients aged 18 or older, regardless of gender, personal historyand probable infection source (hospital or community acquired).

We collected demographic data, the presence of various comorbidities (malignancy, diabetes, chronic renal failure, cardiac, pulmonary, mild/moderate/severe liver pathology, or peripheral vascular, cerebrovascular, haematological diseases, dementia, gastro-duodenal ulcer, presence of concomitant infections). Also possible causes of immunosuppression during the last 2 months prior to the onset (chemotherapy, radiotherapy, corticosteroids, chronic dialysis, surgery- including the type of intervention) and other risk factors (inflammatory bowel disease, colorectal cancer, previous exposure to antimicrobial agents, enteral/parenteral nutrition have been collected. Clinical data (serum albumin, blood leukocyte count, body temperature) with the calculation of the ATLAS Score, recording progress and treatment received, were also considered.

The aetiology was confirmed by the VIDAS ${ }^{\circledR} C$. difficile Toxin $A \& B$ (bioMérieux) test, an ELFA (EnzymeLinked Fluorescent Assay) that detects toxins $A$ and $B$ in fresh stool samples. The ribotyping was performed by 
the Real-Time PCR method from the patients' stool, which allowed the differentiation between the B-toxin and binary toxin, as well as the presumed detection of the strain 027 / NAP / B1.

\section{Results and discussions}

Between J anuary 2018 and December 2018 there were 207 patients admitted to Hospital of Infectious Diseases and Pneumophtisiology Victor Babes Timisoara, diagnosed with Acute Enterocolitis with C. difficile. Of the 207 patients, the nosocomial source was documented in 172 (83\%), and $35(17 \%)$ were cases of no known infection sources (fig. 1a).

The 172 patients represented transfers from other hospitals in the western area of the country, or acquired the infection shortly after admission to a medical / surgical section (fig. 1b).

The age group prevalence was the following: 20-30 years - 4 cases, $30-60$ years - 67 cases, over 60 years -136 cases.

Several prospective and retrospective trials have looked into risk factors, including advanced age, as being contributors to the development and severity of CDI. The three main contributing factors are: exposure to systemic antimicrobial therapy for other infections, exposure to $C$. difficile spores, and the host immune response [8].

As regards to the excessive / unjustified / prolonged or sometimes justified use of antibiotics, clindamycin, ampicillin and cephalosporins were the first antibiotics associated with CDI. The second and third generation cephalosporins, particularly cefotaxime, ceftriaxone, cefuroxime and ceftazidime, are also agents frequently responsible for this condition, and the fluoroquinolones are the most recent atimicrobials to be implicated in hospital outbreaks.

C. difficile is acquired exogenously, most frequently in hospitals or nursing homes, and is carried in the stool of symptomatic and asymptomatic patients.

Atpresent, we are facing an increase in the ICD incidence even after a single dose of antibiotics, more or less justified at home, for various respiratory, intestinal or urinary pathologies. There is sometimes even the tendency of self-

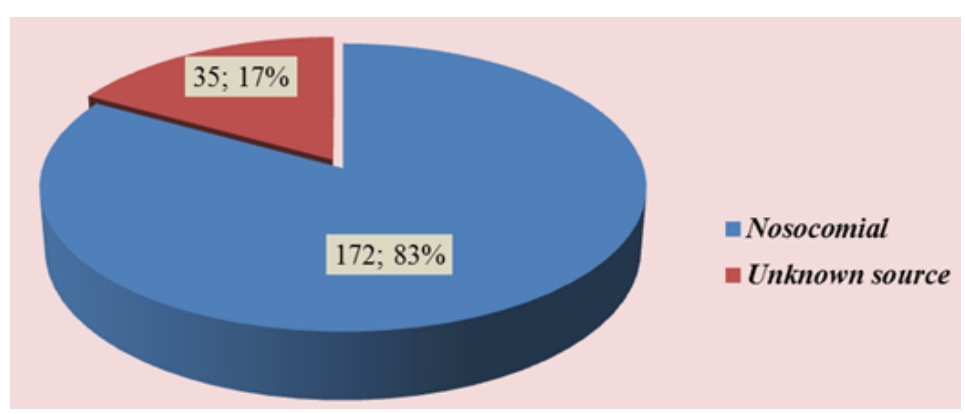

Fig. 1a. Distribution of patients according to the source of the infection

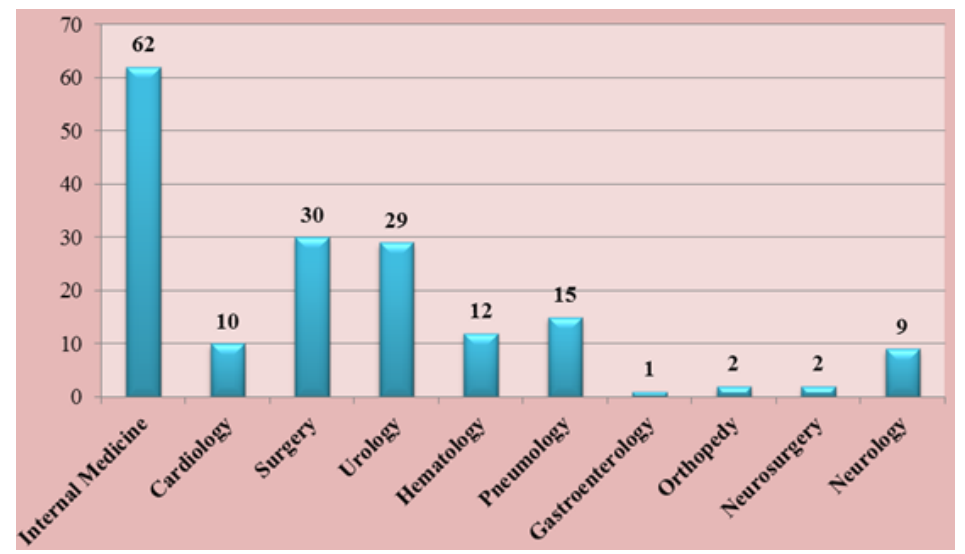

administering an effective antibiotic from the medical history of the patient or of his relatives. Other patients use antibiotics for other purposes than antibacterial, so some elderly people regularly use these medications to maintain the health of some organs.

The physicians from the specialized outpatient clinics should receive periodic reports from the bacteriology laboratories they work with, on the phenomena of antimicrobial resistance within a community. This could reduce the prescription of ineffective antibiotics on pathogenic bacteria, but bactericidal for normal bacterial flora, either to be replaced by opportunistic flora, or to adapt to become carriers of resistance genes.

Of the 207 patients hospitalized with ICD, 151 (73\%) received prior antibiotic treatment. According to the graphic, the alarming increase of unusual antibiotic use at home can be noticed, and can lead to ICD, even without another predisposing favoring factor (fig. 2).

All of the 207 patients included in the study presented the Clostridium A / B, A or B positive positive toxin. Ribotyping was positive in 18 cases. The patients represented transfers from different clinical hospital departments in the western part of the country. Binary toxin with strain 027 was identified in all cases (fig. 3).

Regarding the department where the patients come from, it is interesting to note that most cases come from Internal Medicine (30\% of the total number of cases). Explanations can be multiple:

-the associated pathology is complex: patients with high blood pressure, ischemic heart disease, heart failure, etc., considered as risk factors; from our analysis patients with cardiac pathology represented an important percentage of the total number of cases; in the diagnosis and treatment algorithm of these patients there are many elements to consider: on the one hand the chronic treatment of the underlying disease and on the other hand managing the dehydration syndrome of various degrees sometimes reached up to the hypovolemic shock.

-all cases of $C$. difficile enterocolitis are transferred to the infectious disease department compared to other countries, where each medical or surgical department 


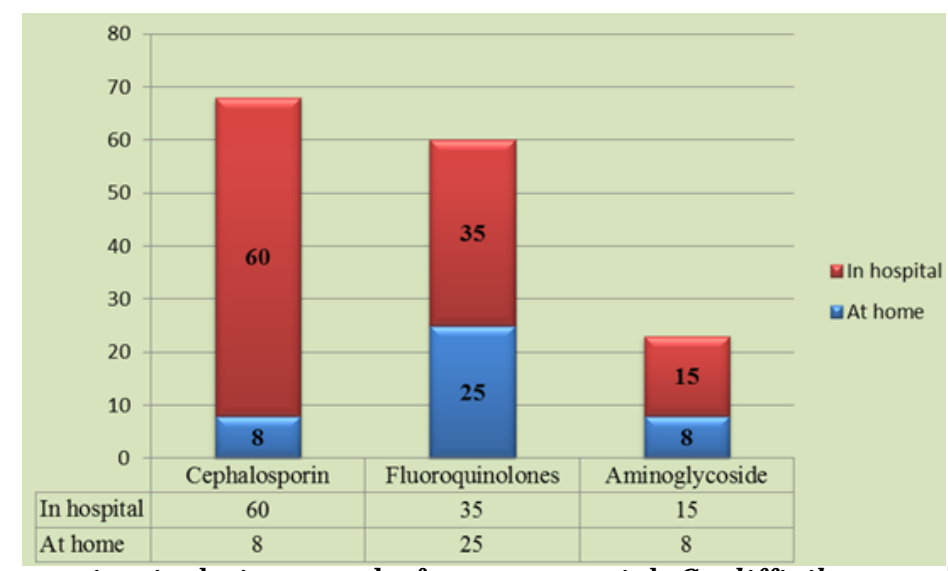

contains isolation wards for nosocomial C. difficile enterocolitis;

We would have expected most cases to come from surgical departments - where complex interventions, antibiotic pre-and / or post-operative treatments are taking place.

Patients also transferred from the Urology departments (14\% of the total number of cases), where due to the current modern possibilities of investigation and treatment for the various pathologies in the renal sphere (especially for the tumoral processes) they require antibiotic associations for pre or post interventions, which has led to an increase in the number of cases of $C$. difficile enterocolitis in recent years.

Many cases originated from the Hematology Department ( $6 \%$ of all cases), partlyjustified due to decreased immunity of these patients, complex therapy with many antimicrobials and immunosuppressive regimens); interestingly, many cases have also been transferred from the Pneumology, where there are patients with complex pathology: COPD, respiratory failure, pulmonary fibrosis, corticosteroid therapy, immunosuppressive therapy, modern treatments and many antibiotic regimens.

Of the 18 ribotyped patients, one showed a mild form of ICD, 14 patients had an average disease, and 3 patients had a fulminant form. The 3 cases with severe ICD and ribotype 027 died after an average of 5 days of hospitalization.

Robinson and colleagues (2014) tested the hypothesis that vegetative cells of hypervirulent $C$. difficile strains could outcompete endemic strains for niche space. Four ribotype 027 clinical isolates and clinical isolates of four other strains (001, 002, 014 and 053) were pairwise tested in human fecal bioreactors and in a humanized microbiota mouse model. Ribotype 027 strains outcompeted endemic strains both in vitro and in vivo and the authors postulated that this competitive advantage is key to the overrepresentation of 027 in recent outbreaks [9-12].
Fig. 2. Antibiotic therapy used prior to admission

The ATLAS score was developed to predict cure and recurrence due to $\mathrm{CDI}$. It is composed of 5 components: Age, Temperature, Leukocytes, Albumin, and Systemic antibiotics, and it was to be used at the bedside at the time of diagnosis of CDI; each variable was assigned 0-2 points [13]. We evaluated the utility of this scoring system as a predictor of mortality due to CDI.

Of the 207 hospitalized patients, it was possible to see in the age group of over 60 years, 15 cases of ribotype 027 which in conjunction with the Atlas score showed a moderate / severe form of disease (fig. 4).

The ATLAS score also maintains its usefulness for follow- up, respectively $97 \%$ of patients at 60 days post ICD had a favorable evolution.

Regarding the therapeutic regimen chosen for the hospitalized patients, 58 patients $(28 \%)$ received Metronidazole treatment for 14 days, 93 patients (45\%) received Vancomycin 14 days, 34 (16\%) Metronidazole and Vancomycin 14 days and 22 (11\%) Metronidazole in combination with Rifamycine for 21 days (fig. 5).

By correlating the ribotype with the chosen treatment regimen, 4 patients classified as mild initiated on Metronidazole treatment relapsed, subsequently evolving later on a average form of disease. Strain 027 proved by ribotyping, which subsequently responded favorably to Vancomycin treatment.

Studies in the recentyears have shown superior efficacy of Vancomycin compared to Metronidazole, in addition, from our clinical experience, patients treated with Vancomycin from the first episode have a lower risk of recurrence.

Current guidelines recommend initiating Vancomycin treatment, including mild illness, from the first ICD episode.

In this study, 3 patients from the studied sample had a severe infection, corroborated with the 027 ribotype and the ATLAS score, which did not respond favorably to specific treatment.

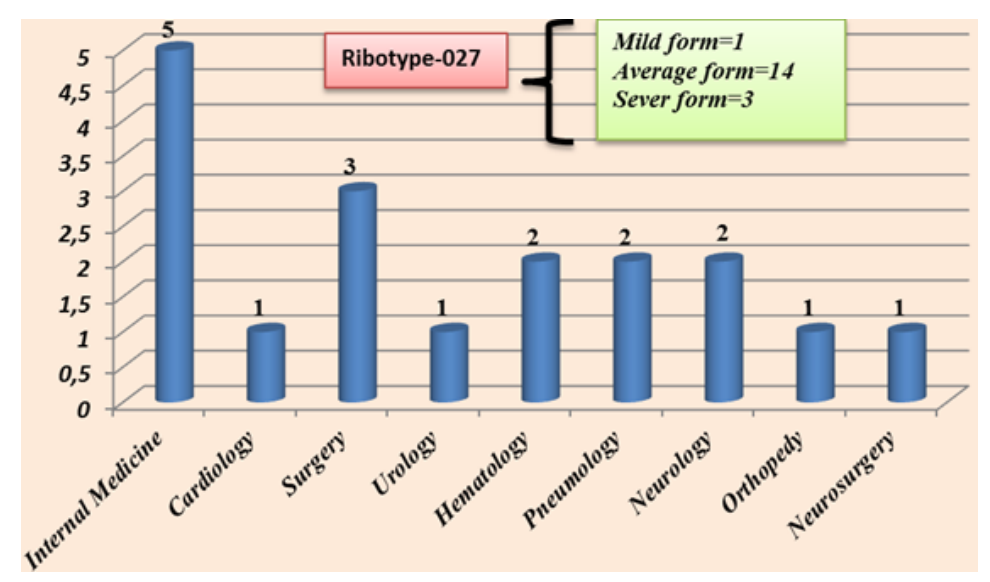

Fig. 3. CDI ribotype correlated with the clinical department 


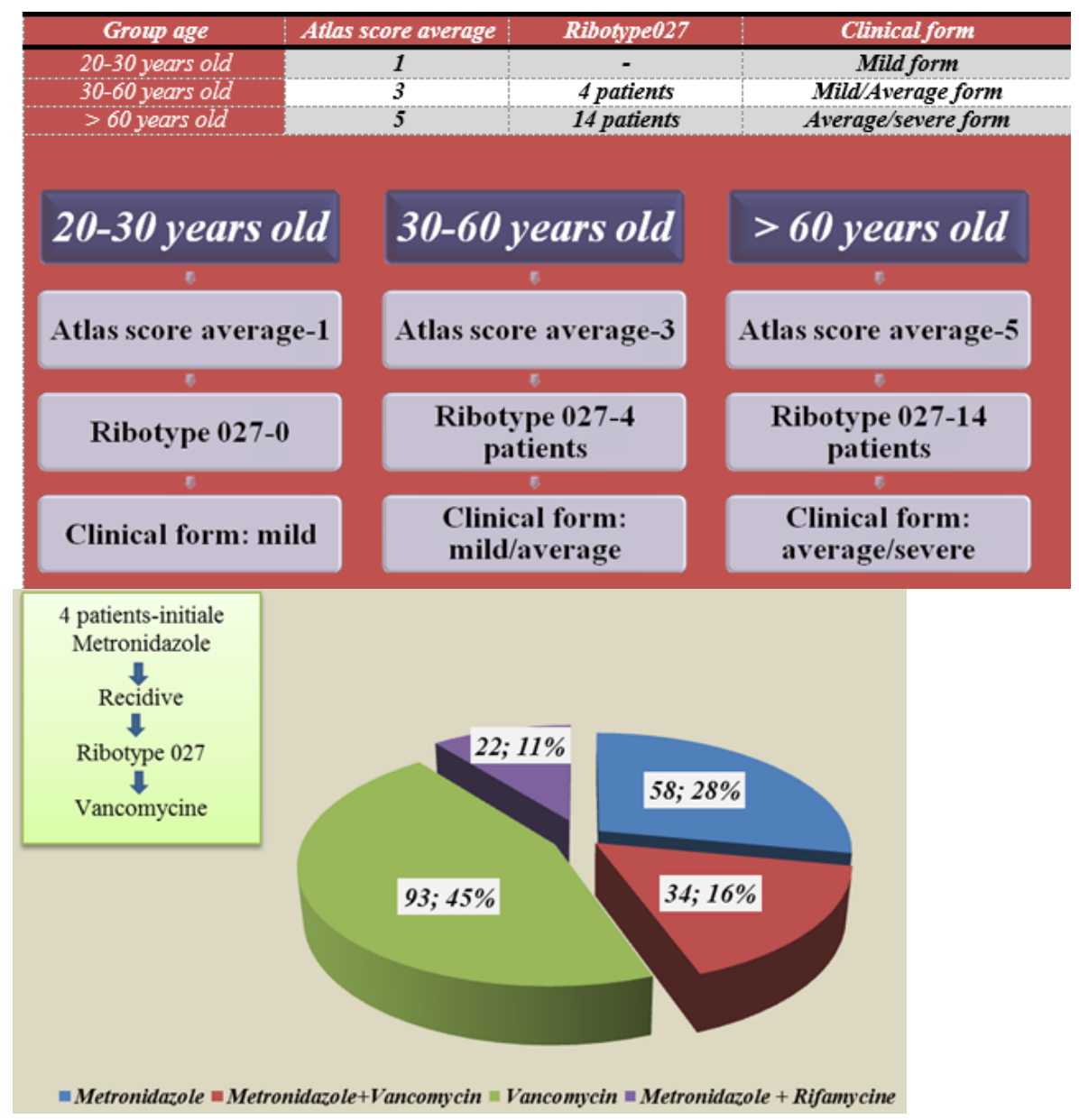

Fig. 4. CDI ribotype correlated with clinical form and ATLAS score

Fig. 5. CDI treatment regimen

ECDC proposed the diagnostic algorithm for the $C$. difficile infections, adapted to the ESCMID guideline [14].

In our hospital it is not possible to perform the diagnosis according to this algorithm, which is a limitation of this study. Another limitation could also be represented by the impossibility of performing genetic tests for all the patients, due to technical reasons.

\section{Conclusions}

C. difficile infection remains a major issue, partly because of the high mortality rate, and on the other hand because of the high cost of hospitalization. In our hospital, the number of $C$. difficile infection has increased significantly over the past year, with about 100 cases compared to the last year.

The virulence of the strains 027 demonstrates once again the importance of ribotyping even in the mild forms of disease. Of the patients with the ribotype $027,14 \mathrm{had}$ an average form of the disease and 3 patients had a severe form of the disease.

Most cases came from the Department of Internal Medicine, at the opposite are situated Neurosurgery, Orthopedics and Gastroenetrology departments.

As expected, the first treatment regimen selected was Vancomycin, however a significant number of cases received Metronidazole as the first choice.

Of course these data are just observations from our casuistry. Certain further investigation is needed. In addition to the Atlas score, we believe that other associations of risk factors should be considered, and it would also be useful and beneficial to use probabilistic mathematical calculations with the contribution of informaticians.

\section{List of abreviations}

CDI - Clostridium difficile infection

USA-United States of America

ECDC- European Centre for Diseases Prevention and Control

ESCMID-European Society of Clinical Microbiology and Infectious Diseases C. difficile-Clostridium Difficile

COPD-Chronic obstructive pulmonary disease

\section{References}

1. STARR, J. M., CAMPBELL, A., RENSHAW, E., POXTON, I. R. \& GIBSON, G. J .Spatio-temporal stochastic modelling of Clostridium difficile. J. Hosp. Infect., 49-56 (2009).

2. RUBIN, M. A. ET AL. A Simulation-Based Assessment of Strategies to Control Clostridium Difficile Transmission and Infection. PLOS ONE 8, e80671 (2013).

3. CODELLA, J., SAFDAR, N., HEFFERNAN, R. \& ALAGOZ, O. An Agent-based Simulation Model for Clostridium difficile Infection Control. Med. Dec. Making 35, 211-29 (2015).

4. LANZAS, C. \& DUBBERKE, D. Effectiveness of screening hospital admissions to detect asymptomatic carriers of Clostridium difficile: a modeling evaluation. Infect. Control Hosp. Epidemiol.

5. MARKELOV A, LIVERT D, KOHLI H. Predictors of Fatal Outcome after Colectomy for Fulminant Clostridium difficile Colitis: A 10-Year Experience. Am Surgeon. 2011;7(8):977.

6. LANZAS, C., DUBBERKE, E. LU, Z., RESKE, K. \& GRÖHN, Y.Epidemiological Model for Clostridium difficile Transmission in Healthcare Settings. Infect. Control Hosp. Epidemiol. 32, 553-561 (2011).

7. YAKOB, L., RILEY, T., PATERSON, D. \& CLEMENTS, A. Clostridium difficile exposure as an insidious source of infection in healthcare settings: an epidemiological model. BMC Infect. Dis. 13, 376 (2013). 8. OWENS RC, JR, DONSKEY CJ, GAYNES RP, LOO VG, MUTO CA. Antimicrobial-Associated Risk Factors for Clostridium difficile Infection. Clin Infect Dis. 2008;46(S1):S19-S31. doi: 10.1086/ 521859.

9. ROBINSON, C. D., AUCHTUNG, J. M., COLLINS, J. \& BRITTON,

R. Epidemic Clostridium difficile strains demonstrate increased 
competitive fitness over non-epidemic isolates. Infect. Immun. 82, 2815-25(2014).

10. YAKOB, L., RILEY, T. V., PATERSON, D. L., MARQUESS, J. \& CLEMENTS, A. C. A.Assessing control bundles for Clostridium difficile: a review and mathematical model. Emerg Microbes Infect 3 , e43; 10.1038/emi.2014.43(2014).

11. WALKER, A. S. ET AL. Characterisation of Clostridium difficile Hospital Ward-Based Transmission Using Extensive Epidemiological Data and Molecular Typing. PLoS Med 9, e1001172 (2012).

12. KERMACK, W. O.\& MCKENDRICK, A. G. A Contribution to the Mathematical Theory of Epidemics. Proc. Roy. Soc. A 115, 700-721 (1927).
13. LAZA R, JURAC R, CRIaAN A, ET AL. Clostridium difficile in western Romania: unfavourable outcome predictors in a hospital for infectious diseases. BMC Infectious Diseases (2015) 15:141.

14. TSCHUDIN-SUTTER S, ET ALL; COMMITTEE, Guidance document for prevention of Clostridium difficile infection in acute healthcare settings, Clin Microbiol Infect. 2018 Oct;24(10):1051-1054. doi: 10.1016/ j.cmi.2018.02.020. Epub 2018 Mar 2.

Manuscript received: 22.11 .2018 